\title{
Microfiber Coupler Based Biosensor Incorporating a Layer of Gold Nanoparticles with Improved Sensitivity
}

\author{
Yuliya Semenova \\ Technological University Dublin, yuliya.semenova@tudublin.ie \\ Lin Bo \\ Technological University Dublin \\ Pengfei Wang \\ Technological University Dublin, pengfei.wang@tudublin.ie
}

See next page for additional authors

Follow this and additional works at: https://arrow.tudublin.ie/engschmanart

\section{Recommended Citation}

Semenova, Y., Farrell, G., \& Wang, P. (2014). Microfiber Coupler Based Biosensor Incorporating a Layer of Gold Nanoparticles with Improved Sensitivity, Proceedings of SPIE - The International Society for Optical Engineering, vol. 91578T-1. doi: 10.1117/12.2059662

This Article is brought to you for free and open access by the School of Manufacturing and Design Engineering at ARROW@TU Dublin. It has been accepted for inclusion in Articles by an authorized administrator of ARROW@TU Dublin. For more information, please contact arrow.admin@tudublin.ie, aisling.coyne@tudublin.ie, gerard.connolly@tudublin.ie.

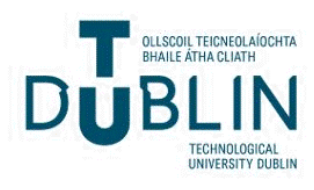




\section{Authors}

Yuliya Semenova, Lin Bo, Pengfei Wang, Furong Tian, Hugh J. Byrne, and Gerald Farrell 


\title{
Microfiber coupler based biosensor incorporating a layer of gold nanoparticles with improved sensitivity
}

\author{
Yuliya Semenova*a ${ }^{*}$ Lin Bo ${ }^{a}$, Pengfei Wang ${ }^{\mathrm{a}}$, Furong Tian ${ }^{\mathrm{b}}$, Hugh Byrne ${ }^{\mathrm{b}}$ and Gerald Farrell ${ }^{\mathrm{a}}$ \\ ${ }^{a}$ Photonics Research Centre, Dublin Institute of Technology, Kevin St, Dublin 8, Ireland; \\ ${ }^{\mathrm{b}}$ FOCAS Research Institute, Dublin Institute of Technology, Camden Row, Dublin 8, Ireland
}

\begin{abstract}
We studied the effect of a star-shaped gold nanoparticles layer coated on the surface of the microfiber coupler (MFC) on the sensitivity of the embedded MFC biosensor. It is shown that deposition of the layer of star-shaped gold nanoparticles on the MFC sensor surface results in a significantly increased spectral shift (on average $3.05 \mathrm{~nm}$ shift compared to a 1.08 $\mathrm{nm}$ shift per layer of electrolyte for the sample without the nanoparticles layer). In addition, introducing the nanoparticle layer results in the decrease of the transmission power; measurement of the changes in transmission also could be used as a means for the sensor interrogation.
\end{abstract}

Keywords: Optical fiber sensor, microfiber coupler, nanoparticles, label-free immunosensor.

\section{INTRODUCTION}

Subwavelength diameter optical fibers, or simply "microfibers" have been intensively studied for various photonic applications and in particular for use in various optical sensors. Compared with other types of fiber-optic sensors, e.g. long-period gratings [1], fiber Bragg gratings [2], side-polished fibers (D-type fibers) [3], and cladding-removed fibers [4], microfibers have a number of advantages, including very large evanescent fields, strong mode confinement and micro-scale diameters. The large fractional power of the evanescent wave surrounding the fiber waist is sensitive to environmental changes, and thus microfibers are shown to have better sensitivity, faster response combined with a compact size, thus offering opportunities for new biosensing techniques [5]. Recently we reported a label-free immunosensor based on a microfiber coupler (MFC) structure embedded in polymer and experimentally demonstrated its performance using a fibrinogen antigen-antibody pair [6]. One common problem in development of such label-free immunosensors is the need to ensure adherence of antibodies to the MFC surface which in turn allows for the efficient binding of the corresponding antigen resulting in improved sensitivity. A number of research groups have reported pioneering results related to potential of nanoscale sensors for label-free detection based on utilizing noble metal nanoparticles [7-9].

It is well known that noble metal nanoparticles, such as gold and silver, exhibit a strong optical extinction at visible and near-infrared wavelengths and generate localized surface plasmon resonance (LSPR) phenomenon which is sensitive to the refractive index (RI) of the surrounding medium [10]. Moreover, it has been shown that the biomolecular interactions on the nanoparticle surface can change the evanescent field distribution. For example, the authors in [11] demonstrated that in order to impart selectivity to a fiber-optic end-face sensor based on LSPR, a material with affinity to the desired target-substance can be attached to the nanoparticles. In this work, we investigated the possibility of utilizing the properties of gold nanoparticles coated on the surface of the MFC in order to improve the sensitivity of our recently proposed MFC immunosensor. Here we experimentally demonstrate enhancement of the sensor performance after coating the MFC surface with a layer of star-shaped gold nanoparticles. By monitoring the spectral shift of the MFC based sensor in the wavelength domain, the sensor is capable of detecting alternate layers of poly (allylamine hydrochloride) (PAH) and poly (styrene sulfonate) (PSS) which were grown on the MFC surface using the layer-by-layer $(\mathrm{LbL})$ assembly technique. Deposition of the layer of star-shaped gold nanoparticles with a diameter of $\sim 120 \mathrm{~nm}$ on the MFC sensor surface results in a significantly increased spectral shift corresponding to each polyelectrolyte layer

* Corresponding author: yuliya.semenova@dit.ie; 
(on average $3.05 \mathrm{~nm}$ shift versus $1.08 \mathrm{~nm}$ shift per layer of electrolyte for the sample without the nanoparticle layer). In addition, introducing the nanoparticles layer results in the decrease of the transmission power; measurement of the changes in transmission also could be used as a means for the sensor interrogation.

\section{MFC SENSOR FABRICATION AND EXPERIMENT}

An MFC was fabricated by fusing and tapering of two single-mode optical fibers SMF28 together using a microheater brushing and tapering technique [12]. The MFC was weakly fused and the minimum tapered diameter of each fiber was approximately $2.5 \mu \mathrm{m}$. The experimental setup for MFC fabrication is shown in Figure 1 . As the diameter of the fiber coupler reduces to several microns, the fiber coupler reaches a state where it displays a strong evanescent field (due to the wavelength-scale waist diameter) and where the transmission spectrum shows a semi-periodically resonant behavior for each of the output ports due to the even-odd mode interference between the fused fibers.

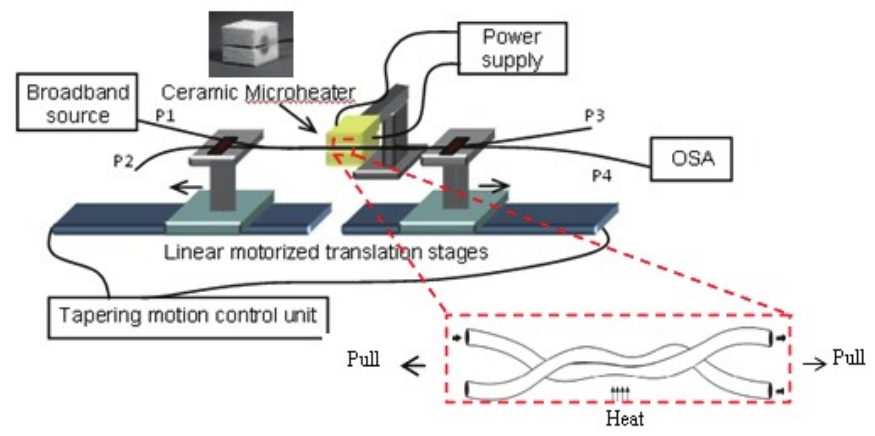

Figure 1. MFC fabrication setup based on the microheater brushing technique.

The fabricated MFC was partially embedded in a low RI UV curable polymer substrate to improve the mechanical stability of the sensing system as illustrated in Figure 2(a). One input port of the fiber taper was connected to a superluminescent diode (SLD) (Thorlabs), with a wavelength range of 1500-1600 nm and one of the outputs of the fiber taper was connected to the Optical Spectrum Analyser (OSA) (86142B, Agilent) where the output transmission spectra were recorded.

Alternate layers of poly (allylamine hydrochloride) (PAH) and poly (styrene sulfonate) (PSS) were then grown on the MFC surface from the water solutions with concentrations of $5.5 \mathrm{mg} / \mathrm{ml}$ for each polyelectrolyte, using the thin film Layer-by-Layer (LbL) assembly technique (inset of Figure 2(a)). LbL assembly works by depositing alternating layers of oppositely charged materials with a wash step in between. Corresponding transmission spectra are shown in Figure 2(b).
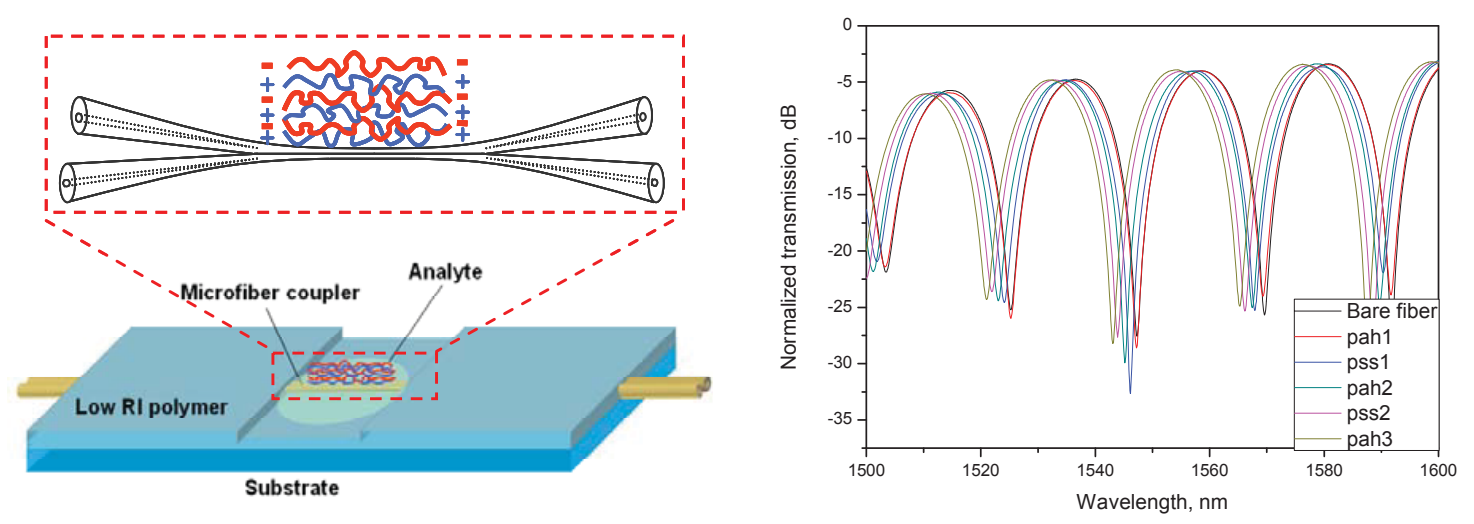

Figure 2. (a) Schematic diagram of the embedded MFC and illustration of PAH/PSS adsorption on MFC surface; (b) Transmission spectra of the MFC recorded after deposition of each polyelectrolyte layer. 
As it can be seen from the figure each consecutive layer of the PAH/PSS results in a blue shift in the transmission spectrum of the MFC. The average shift was estimated as $\sim 1.08 \mathrm{~nm}$ per layer of the polyelectrolyte.

\section{PREPARATION OF THE MFC SAMPLE WITH NANOPARTICLES LAYER}

The gold nanostars for our experiment were prepared in aqueous phase via the surfactant-directed, seed-mediated growth method. Growth solution was prepared by adding $0.20 \mathrm{ml}$ of $0.01 \mathrm{M} \mathrm{HAuCl}$ : $4 \mathrm{H} 2 \mathrm{O}$ to $4.5 \mathrm{ml}$ of $0.1 \mathrm{M} \mathrm{CTAB}$ in a plastic test tube while gently mixing. To this solution, $0.030 \mathrm{ml} 0.01 \mathrm{M} \mathrm{AgNO3}$ was added. After mixing, the color of the solution becomes brownish yellow. Then, $0.032 \mathrm{ml}$ of $0.1 \mathrm{M}$ ascorbic acid was added, resulting in a colourless solution. Finally, $0.01 \mathrm{ml}$ of the $10 \mathrm{~nm}$ gold colloid suspension was added. After gentle mixing, the solution was kept in a water bath at room temperature undisturbed for 3 hours. The eventual blue-purple colour of the growth solution indicated nanostar formation. Nanostars were then washed three times in a centrifuge at 4500 RPM for 15 minutes.

The MFC sample was fabricated using the same fabrication steps and similar geometrical parameters $(\sim 2.5 \mu \mathrm{m}$ fiber waist diameter for each fiber) as above. In order to immobilize the nanoparticles, two bilayers of PAH and PSS, followed with a layer of PAH were assembled on the MFC surface (as shown in Fig. 2 b). Then the nanoparticles were deposited on top of this (PAH/PSS $)_{2} \mathrm{PAH}$ multilayer. The nanoparticles were deposited from a water suspension with a concentration of approximately $6 \times 10^{8}$ particles $/ \mathrm{ml}$. The average size of the gold nanostars was about $120 \mathrm{~nm}$. After the deposition of the nanoparticles layer the MFC surface was again washed with water.

At the next step the process of growth of alternate layers of poly (allylamine hydrochloride) (PAH) and poly (styrene sulfonate) (PSS) by the LbL assembly technique was repeated as described in the previous section with a wash step in between.

\section{EXPERIMENTAL RESULTS AND DISCUSSION}

Figure 3 shows corresponding transmission spectra recorded after each experimental step.
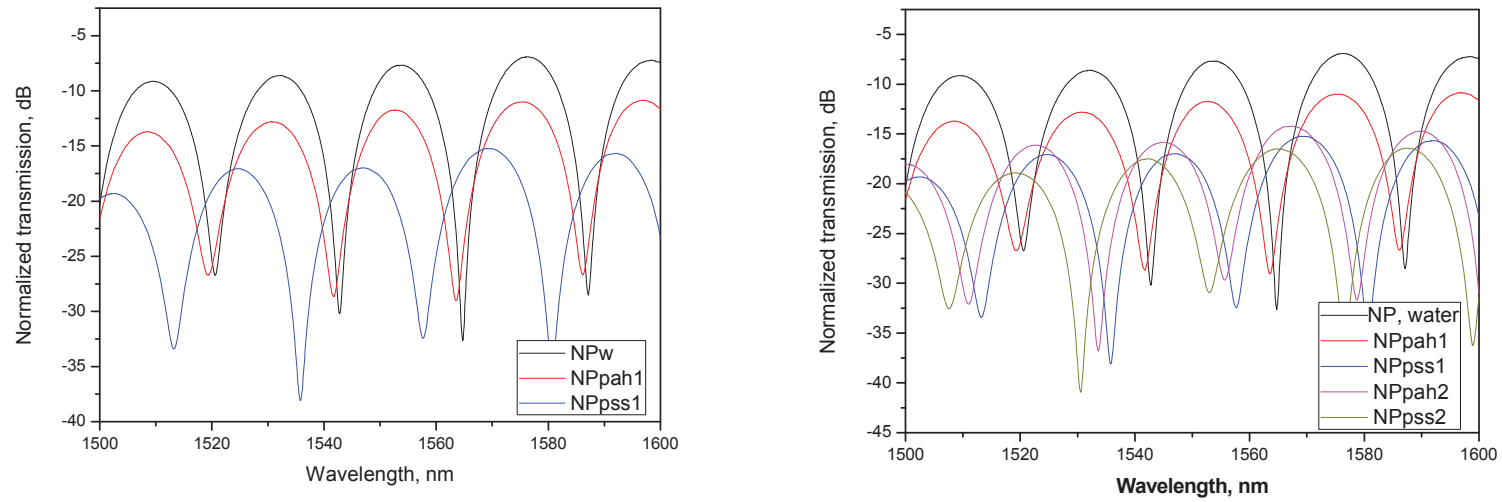

Figure 3. Transmission spectra for the MFC with a layer of star-shaped gold nanoparticles and various layers of polyelectrolytes: (a) bare fiber with nanoparticles, first bi-layer of polyelectrolytes; (b) transmission spectra after the second bi-layer deposition.

As it can be seen from the Figures, after the introduction of the gold nanoparticles layer the spectral blueshift in the MFC spectrum corresponding to each polyelectrolyte layer is significantly increased. It should be noted that the values of the spectral shifts corresponding to the deposition of consecutive layers vary significantly for different layers and tend to increase, for example, an average spectral shift corresponding to the deposition of PAH1 layer is estimated about $1 \mathrm{~nm}$, 
PSS1 - $5.5 \mathrm{~nm}, \mathrm{PAH} 2$ - $2.55 \mathrm{~nm}$ and PSS2 - $3.05 \mathrm{~nm}$. In addition, introduction of the nanoparticles layer results in the decrease of the overall transmission power level of the coupler, which could be used for the MFC interrogation.

\section{CONCLUSION}

In summary, we studied the effect of a star-shaped gold nanoparticles layer coated on the surface of the microfiber coupler (MFC) on the sensitivity of the embedded MFC biosensor. It is shown that deposition of the layer of star-shaped gold nanoparticles on the MFC sensor surface results in a significantly increased spectral shift. An average spectral shift corresponding to the deposition of a single polyelectrolyte layer is estimated as circa $3.05 \mathrm{~nm}$ compared to a circa $1.08 \mathrm{~nm}$ spectral shift per layer of electrolyte for the sample without the nanoparticles. The presented preliminary results demonstrate a potential for the embedded MFC with a layer of nanoparticles to work as a reliable sensor with an improved sensitivity for an extensive range of applications which are based on the detection of sequential adsorption of chemical compounds or specific binding between biomolecules. Further research is ongoing to develop an understanding of interactions between the molecules on the surface of the MFC sensor.

\section{ACKNOWLEDGEMENT}

Lin Bo would like to thank the International Centre for Graduate Education in micro \& nano Engineering (ICGEE) for her PhD funding support.

\section{REFERENCES}

[1] Y.-P. Wang, L. Xiao, D. N. Wang, and W. Jin, "Highly sensitive long-period fiber-grating strain sensor with low temperature sensitivity," Opt. Lett. 31(23), 3414-3416 (2006).

[2] S. Maguis, G. Laffont, P. Ferdinand, B. Carbonnier, K. Kham, T. Mekhalif, and M.-C. Millot, "Biofunctionalized tilted fiber Bragg gratings for label-free immunosensing," Opt. Express 16(23), 1904919062 (2008).

[3] S.-M. Tseng and C.-L. Chen, "Side-polished fibers," Appl. Opt. 31(18), 3438-3447 (1992).

[4] A. Abdelghani and N. Jaffrezic-Renault, "SPR fibre sensor sensitised by fluorosiloxane polymers," Sens. Actuators B, 74(1-3), 117-123 (2001).

[5] F. Xu, P. Horak and G. Brambilla, "Optical microfiber coil resonator refractometric sensor,” Opt. Express 15, 7888-7893 (2007).

[6] L. Bo, C. C. O’Mahony, Y. Semenova, N. Gilmartin, P. Wang and G. Farrell, "Microfiber coupler based labelfree immunosensor," Optics Express, in press.

[7] M. Rani, N. K. Sharma, V. Sajal, "Localized surface plasmon resonance based fiber optic sensor with nanoparticles", Opt. Communications, 292, 92-100 (2013).

[8] H.-Y. Lin, C.-H. Huang, G.-L. Cheng, N.-K. Chen and H.-C. Chui, "Tapered optical fiber sensor based on localized surface Plasmon resonance”, Opt. Express 20, 21693-21700 (2012).

[9] P. Bhatia, P. Yadav, B. D. Gupta, "Surface Plasmon resonance based fiber optic hydrogen peroxide sensor using polymer embedded nanoparticles", Sens. Actuators B, 182, 330-335 (2013).

[10] T.-C. Chang, C.-C. Wu, S.-C. Wang, L.-K. Chau, and W.-H. Hsieh, "Using a fiber optic particle plasmon resonance biosensor to determine kinetic constants of antigen-antibody binding reaction", Anal. Chem. 85, 245-250 (2013).

[11] A. R. Camara, P. M. P. Gouvêa, A. C. M. S. Dias, A. M. B. Braga, R. F. Dutra, R. E. de Araujo, and I. C. S. Carvalho, "Dengue immunoassay with an LSPR fiber optic sensor", Opt. Express 27023-27031 (2013).

[12] G. Brambilla, V. Finazzi, and D. Richardson, "Ultra-low-loss optical fiber nanotapers,” Opt. Express 2258-2263 (2004). 